\title{
Diagnostik in der ärztlichen Praxis, nach Samuel Gottlieb Vogel (1750-1837)
}

Aus dem Medizinhistorischen Institut der Universität Basel

(Vorsteher: Prof.Dr.med.H.Buess)

Von cand.med. Jonas Landmann

Ich möchte an dieser Stelle Herrn Prof. Dr. H. Koelbing, jetzt in Zürich, für die Anregung zum Thema und seine immer liebenswürdig gewährte Hilfe aufrichtig danken.

I. BIOGRAPHIE UND BIBLIOGRAPHIE

1. Vogel, sein Leben und seine Zeit

Samuel Gottlieb Vogel wurde am 12.März 1750 in Erfurt geboren. Sein Vater, Rudolf Augustin Vogel (1724-1774), praktizierender Arzt in Erfurt, wurde 1753 als außerordentlicher Professor nach Göttingen, an die Universität Hallers, berufen. Er muß ein vielseitig belesener und forschender Gelehrter gewsen sein, aber ein konservativer Systematiker, der von neuen Errungenschaften, die sich nicht auf ein System zurückführen ließen, nichts wissen wollte. Nicht nur den medizinischen Wissenschaften widmete er seine Arbeitskraft, auch Heilquellenlehre, Chemie und Mineralogie fesselten seine Aufmerksamkeit. Er hat sich besonders auch dadurch einen Namen geschaffen, daß er die erste rein medizinische Zeitschrift in deutscher Sprache, die Neue medizinische Bibliothek (vgl. 51) begründet und über zwanzig Jahre lang redigiert hat. 1760 wurde er zum Ordinarius ernannt und schuf, sicher nach Boerhaaveschem Vorbild, ein «collegium clinicum ».

In dieser lebendigen und von der Medizin getragenen Atmosphäre also wuchs S.G.Vogel auf. Er war ein frühreifes, fleißiges Kind, und schon 15 jährig begann er in Göttingen sein Medizinstudium. 1771 beendete er seine Ausbildung und erlangte am 30.Dezember mit seiner Dissertation De Lithophago et Polyphago Ilefeldae nuper mortuo et dissecto seinen Doktor der Medizin.

Nach Abschluß seiner Studien war er erst einmal in Göttingen als praktischer Arzt tätig, bis er sich 1776 für kurze Zeit habilitierte. Er zog sich aber sehr bald vom Universitätsbetrieb zurück und ging vorerst wieder in die Praxis nach Ratzeburg, wo er mehrere kleine Schriften verfaßt hat. Er fand dort ein weites Tätigkeitsfeld und bald auch gehörige Anerkennung; 
so wurde er z.B. als Dank dafür, daß er einen ehrenvollen Ruf nach Pavia aus Vaterlandsliebe und Bescheidenheit abgelehnt hatte, 1784 vom König von England zum Hofmedicus ernannt - ein Ehrentitel, der schon seinen Vater ausgezeichnet hatte. Die Nachfolge des berühmten Waadtländers Tissot, der auf die Bemühungen Kaiser Joseph des Zweiten 1780 im österreichischen Pavia den medizinischen Lehrstuhl übernommen hatte, hat dann J.P.Frank 1786 angetreten. Im Jahre 1832 wurde Vogel vom König von Bayern geadelt. Seit 1789 hatte er den medizinischen Lehrstuhl in Rostock inne.

Welche Strömungen und Ideen haben nun diese Jahrzehnte erfüllt?

Eine Vielzahl von sehr mannigfaltigen naturwissenschaftlichen Erkenntnissen haben sich im 16. und 17. Jahrhundert angesammelt. Je weiter diese Entwicklungen fortschritten, desto unglaubwürdiger und zweifelhafter wurde das althergebrachte medizinische Lehrgebäude des Galen. Was von diesem neuen Grundlagenwissen in die klinische Medizin Eingang gefunden hat, spiegelt sich in den Theorien der Iatrophysiker und Iatrochemiker wider, und es ist sehr wenig Brauchbares dabei: der wirklich bedeutende diagnostische Beitrag des 17.Jahrhunderts war die klinische Beobachtung eines Sydenham und anderer, die damit wieder bei Hippokrates Anschluß gesucht haben. Der große Unterschied liegt allerdings darin, daß nun das Hauptgewicht des Interesses bei der Krankheit lag und nicht wie im Altertum beim kranken Menschen. «Hippokrates schreibt Krankengeschichten, Sydenham dagegen die Geschichte von Krankheiten» (50). Daß diese klinisch-praktischen Observationen Fortschritte gebracht haben, liegt teils am geradezu pedantischen Beobachten und Schildern der Krankheiten am Krankenbett, ohne sich lange bei Theorien aufzuhalten, teils aber auch am Miteinbeziehen pathologisch-anatomischer Anschauungen. Wenn Morgagni auch erst ein Jahrhundert später sein berühmtes Werk veröffentlicht hat, so war er doch lange nicht der erste, der sich diesem wichtigen Zweig der Medizin zugewendet hat.

Auf der Schwelle zum 18. Jahrhundert meldeten sich als Antwort auf die sachfremden iatrophysikalischen und -chemischen Spekulationen vitalistische Anschauungen kraftvoll zu Wort. Der Pietist Stahl hat seine Lehre vom Animismus verkündet, in der er den somatischen und den psychischen Bereich, den Körper allen seelischen Einflüssen unterordnend, zu vereinen suchte.

Das Lebensgefühl und die barocke Liebe zu Spekulation über das Absolute und Universale, über Gott und Natur, vor allem über die Metaphysik, 
haben noch lange dieses Jahrhundert beherrscht. So ist es paradox, daß in dieser Zeit der medizinischen Systeme derjenige der erfolgreichste Arzt war, der keine Theorie hatte: Boerhaave, sicher der einflußreichste Kliniker und bedeutendste Lehrer seiner Zeit, anerkannte und förderte das, was nützlich, erprobt und erfolgversprechend war.

Eine eindrückliche Antwort auf alle Spekulation hat Haller mit seinen physiologischen Experimenten erteilt. Er hat prinzipiell die gewebespezifischen Eigenschaften von Nerv und Muskel unterscheiden können. Brown hat mit seiner Reiztheorie insofern diese Entdeckungen mißbraucht, als er Charakteristika gewebespezifischer Phänomene zur Erklärung eines ganzen medizinischen Systems herangezogen hat.

Eine nette Anekdote zum Mißbrauch der Nerven in dieser Zeit erzählt Hufeland: früher habe kein Mensch gewußt, daß er Nerven habe ... Da habe vor 40 Jahren Whytt den unseligen Einfall gehabt, ein Buch von den Nerven und ihren Krankheiten zu schreiben, und ein Apotheker, der es gelesen, nachdem er sich über die Zufälle einer Dame lange den Kopf zerbrochen, antwortete ihr nun: es sind die Nerven, Madame. Jetzt wolle alle Welt Nerven haben, und zwar piquiere man sich, scharfe, reizbare, delikate Nerven zu haben (33).

Die Abkehr vom Systematischen zugunsten einzelner Krankheiten und Krankheitseinheiten und ein Zug zum Konkreten dokumentierte das Jahr 1761 in zweifacher Weise, sind doch Morgagnis Sitz und Ursache der Krankheiten und Auenbruggers Inventum Novum erschienen.

Die Wirkung der Auenbruggerschen Schrift war langsam und wenig spektakulär. Die Boerhaave-Schüler van Swieten und de Haen waren die Wiener Medizinpäpste, sehr konservativ und nicht in der Lage, das Geniale an Auenbruggers Methode zu erkennen.

Das aufgeklärte Streben nach einer unvoreingenommenen, aus der Erfahrung erwachsenden Krankheitslehre kam der ärztlichen Praxis in hohem Maße zugute, es wurde konsequent das genaue Beobachten eines jeden Krankheitsverlaufes und seine minutiöse schriftliche Darlegung gefordert, die Sektion als Kontrolle und Belehrung wenn möglich durchgeführt; denn im Gegensatz zum Barock lag dem aufgeklärten Zeitalter das Diesseits am Herzen, man wollte das Beste für das Einzel- und Staatswesen, und nur gesund glaubte man die Glückseligkeit erreichen zu können. So wurde denn auch die Bedeutung der Prophylaxe, Hygiene und Volksaufklärung klar erkannt. Gegen unerträgliche Zustände in Spitälern, Gefängnissen und Armeen wandte sich ein neues soziales Gewissen, das auch für Taubstumme, 
Blinde und Alkoholiker eine adäquate Umgebung und Behandlung zu schaffen gewillt war.

In Frankreich hat der Glaube an eine vernünftige, brauchbare Heilkunde erst nach der Beseitigung der konservativen Fakultäten Wurzeln schlagen können. Die Gedanken der Aufklärung und der Revolution haben ja ohnedies den Menschen als Subjekt wieder entdeckt, so daß die Sozialmedizin weniger betont zu werden brauchte. So hat sich der neue Zeitgeist in einer unbelasteten Umgebung in einem «Anheben der medizinischen Forschung», in der «Entwicklung der experimentellen Methode» (41) auswirken können. Obwohl diese Ansätze der naturwissenschaftlichen Forschung nicht in die Schulmedizin Eingang gefunden haben, hat Paris mit der Berücksichtigung der makroskopischen Pathologie die Vormachtstellung in der Medizin für Jahrzehnte erringen können.

Im Wien der Zeit zwischen den beiden Höhepunkten der ersten und zweiten Wiener Schule und in Deutschland war man unsicher und enttäuscht: da man die rationale Medizin doch nur mit halbem Herzen betrieb, fehlte der Elan, und der Erfolg blieb aus. Man warf sich einmal mehr in die Arme der Philosophie. Schelling, der Exponent der Naturphilosophie, hat nicht so sehr originell Neues gebracht, dafür aber die bereits so einflußreichen wie bekämpften Theorien und Erkenntnisse eines Galvani, Volta, Mesmer oder Cullen und Brown in einem philosophischen Überbau zusammengefaßt und dadurch seiner Zeit eine neue Richtung gewiesen, nach der sie schon gesucht hat. Es ist verständlich, daß das Suchen nach der «Idee hinter der Fassade der äußeren Erscheinung» (48) zum Gegenteil dessen geführt hat, was Morgagni und Auenbrugger mit ihrer Frage «ubi est morbus» (40) angestrebt und nach ihrem Tode erst erreicht haben.

Eine Stelle aus dem Brief an einen Hofrat Römer in Berlin von 1811 möge bezeugen, wie wenig Vogel sich mit der Naturphilosophie hat anfreunden können: «Verwahren Sie ja Ihr Journal vor aller Naturphilosophie. Diese, soweit der Unsinn derselben geht, und insofern sie sich in die Medicin mischt, muß von allen Seiten verfolgt werden. Wenn ich ein mir unbekanntes Buch aufschlage und finde die geringsten Spuren von derselben, so mache ich sicher das Buch sogleich wieder zu und rühre es gewiß nicht wieder an. Ich hoffe, unseres Hofraths Links [von 1792 bis 1811 Professor in Rostock für Naturgeschichte] neuestes Werk: über Natur und Philosophie wird eine bedeutende Revolution veranlassen, wodurch das tolle, abgeschmackte, unverständliche Zeug endlich in die ewige Vergessenheit kommen wird» (18). 
Nun aber endgültig zurück zu Vogels Lebenslauf: 1789 ist das Jahr seiner Berufung nach Rostock. Hier entwickelte er eine vielseitige, ihn ausfüllende Aktivität. Er begründete bald das Seebad Doberan, das die erste Stätte dieser Art in Deutschland war und ihm den Beinamen «Vater des deutschen Seebades» eingetragen hat. In den Sommermonaten war er von jetzt an als Badearzt voll beschäftigt. Literarisch fand diese Seite seines Wirkens in den folgenden Schriften ihren Niederschlag: Über den Nutzen und Gebrauch der Seebäder (Stendal 1794); Baderegeln zum Gebrauch für Badelustige ... (Stendal 1817); Kurze Übersicht der sämtlichen Seebäder in Deutschland (Rostock 1831) und verschiedene Abhandlungen in Baldinger's neuem Magazin für Ärzte und anderen Zeitschriften und Zeitungen.

Eine umfangreiche literarische Produktion bezieht sich auf die Medizin im engeren Sinne. Sein Hauptanliegen war zweifellos die Diagnostik, dargestellt im Krankenexamen (Stendal 1796) und in den Allgemeinen medicinisch diagnostischen Untersuchungen 1. (Stendal 1824) und 2. Theil (Stendal 1831).

Aber auch alle andern Gebiete der Medizin, die Pathologie, Therapie, gerichtliche Medizin, Hygiene und die populäre Medizin sind in seinen Schriften mindestens berührt. Er soll eine ausgedehnte Korrespondenz mit vielen berühmten Zeit- und Fachgenossen geführt haben. Hochbetagt erlag er am 19. Januar 1837 einer Grippe.

\section{2. Überblick über die diagnostischen Schriften}

a) Das Krankenexamen

In der Vorrede zu seinem Krankenexamen erwähnt Vogel die Literatur, wie sie über die allgemeine Diagnostik bereits vorliegt, beurteilt sie als zu wenig umfassend und begründet damit, was ihn zum Abfassen dieser Schrift bewogen hat. Das Studium solcher Werke rechtfertigt seinen Entschluß, der ein wenig verbreitetes Aufgeschlossensein dokumentiert. Gleichzeitig verspricht er dem Leser eine spezielle und detailliertere Anleitung über denselben Gegenstand, also seine rund dreißig Jahre später erscheinenden Allgemeinen medicinisch diagnostischen Untersuchungen.

Die Einleitung des Krankenexamens betont die zentrale Stellung der Krankenuntersuchung in der Medizin. Das Stellen einer Diagnose sei die schwierigste ärztliche Handlung, man müsse sich vor mancherlei Täuschung hüten, aber nichts zeichne einen Arzt mehr aus, als in diesem Punkte richtig vorzugehen. Das erste Kapitel widmet Vogel allgemeinen Anweisungen 
über die Art, wie man zu einer möglichst brauchbaren Anamnese und einem vollständigen Status gelange. Er betont, wie wichtig der affektive Kontakt zwischen Arzt und Patient sei, wie der Arzt aufzutreten, wie er beim Befragen vorzugehen und welche Zurückhaltung er sich aufzuerlegen habe, wenn er einem Patienten Aufschluß über seinen Zustand gebe.

In den folgenden Kapiteln sind die Besonderheiten der Untersuchung der Kinder, Frauen und Geisteskranken aufgeführt.

Im 5. Kapitel beschreibt Vogel kursorisch, im 7. ausführlicher, welche Fragen und Untersuchungen eine Krankheit erhellen. Es ist bemerkenswert, daß er den Gang der Untersuchung in Familienanamnese, persönliche Anamnese, jetziges Leiden und Status praesens gliedert, wenn auch nicht immer mit diesen Bezeichnungen. (Dies rechtfertigt die Einteilung, wie sie unten im Abschnitt III gewählt wurde; sie ist keineswegs der mühsame Versuch, Vogel moderner erscheinen zu lassen, als er war.)

Das 6. Kapitel ist dem Kennenlernen des kranken Individuums gewidmet. Hiebei fällt auf, wie starkes Gewicht Vogel der außerindividuellen Sphäre im soziologischen Sinne als Ursache oder mindestens als beeinflussendem Faktor vieler Krankheiten beimißt.

In den folgenden Kapiteln werden die einzelnen Punkte der Statuserhebung erläutert, steht, wie die einzelnen Befunde zu werten und verwerten seien, sind therapeutische Überlegungen festgehalten, wird die wichtige Kenntnis des Krankheitsverlaufes gewürdigt und die Kausalität der verschiedenen Krankheitsentwicklungen untersucht.

\section{b) Die Allgemeinen medicinisch diagnostischen Untersuchungen}

In der Vorrede zu den Allgemeinen medicinisch diagnostischen Untersuchungen zur Erweiterung und Vervollkommnung seines Krankenexamens zitiert Vogel mehrere, vorwiegend positive Rezensionen des Krankenexamens.

Die Einleitung lobt und begründet das Zusammengehen von Empirismus und Dogmatismus (im Sinne eines sytematischen, theoretischen Wissens) in der Praxis, weil der Kranke mehr durch empirisch geschulte Beobachtung, seine Krankheit mehr durch das Wissen über das Wesentliche auf Grund der theoretisch fundierten Medizin beurteilt werden könne. Vogel hebt die Grenzen hervor, an die die ärztliche Kunst immer wieder stoße; er beurteilt dies insofern positiv, als es den Dünkel der Ärzte dämpfe. In seinen Medicinischen Beobachtungen und Memorabilien aus der Erfahrung (Stendal 1834) steht die in diesem Zusammenhang bemerkenswerte auto- 
biographische Notiz: «Wenn ein Arzt, wie der Verfasser dieses Buches, der Sohn eines gelehrten Arztes und öffentlichen Lehrers, nach guter Vorbereitung und unter fortdauernden fleißigen Studien, sorgsam geleitet an der Hand eines sehr verständigen, vorsichtigen und liebreichen Vaters, fünfzig bis sechzig Jahre lang ... unter den verschiedensten Bedingungen, Verhältnissen und Umständen, fast täglich Kranke gesehen, beobachtet und behandelt hat, selbst auch während dieser Zeit seit 44 Jahren auf einer Hochschule Lehrer seiner Kunst gewesen ist, dann sollte man glauben, derselbe sei nun in die Tiefen der Heilkunst möglichst eingedrungen, könne nach den erforderlichen Forschungen sofort die Natur einer jeden Krankheit erkennen und wisse auf ein Haar, was und wie alles geschehen müsse, um sie nach Möglichkeit gründlich zu heilen. Ein tiefes Bedauern durchdringt mich, gestehen zu müssen, da $\beta$ dem leider! nicht so ist, daß vielmehr kaum ein Tag hingeht, an welchem ich nicht Krankheitsfälle vor mir sehe, die ich mit entschiedener Gewissheit vollständig zu ergründen und zu durchschauen und demnach mit voller Sicherheit zu behandeln mich nicht im Stande befinde.»

Das 1. Kapitel der Allgemeinen medicinisch diagnostischen Untersuchungen nun spricht von den bei der Untersuchung auftauchenden Hindernissen und Schwierigkeiten von seiten des Arztes, des Kranken und der Krankheit. Das 2. Kapitel hebt ausführlich die Bedeutung des Erkennens der ätiologischen Zusammenhänge hervor. Es ist frappant zu lesen, wie klar, wenn auch nicht immer richtig, Vogels Angaben sind, solange sie sich auf morphologische Veränderungen beziehen, die funktionell erklärbar sind, besonders auch gewisse Mißbildungen, wie unkritisch und phantastisch dagegen seine Erklärungen für Infektionskrankheiten und generalisierte Affektionen mit scheinbar nur geringfügigen morphologischen Veränderungen werden.

Im kurzen Schlußkapitel des ersten Bandes deutet Vogel die Kriterien einer einwandfreien Beobachtung für diagnostische wie therapeutische Fragen an.

Der sieben Jahre später erschienene zweite Band schließt inhaltlich nicht direkt an den ersten an. So finden sich im 1. Kapitel wieder generelle Angaben zur Diagnostik, die besonders die Sorgfaltspflicht betonen. Das 2. Kapitel behandelt nicht das «Was», sondern das «Wie» der Diagnostik, was Vogel das «Savoir faire» nennt, den «Schlüssel zu einer weisen Diagnostik». Hier legt er Zeugnis ab von seiner bemerkenswerten Ethik, erläutert anhand von delikaten Beispielen, wie sich ein Arzt auch in unge- 
wohnten Situationen zu verhalten habe und gibt schließlich einige Winke, wie der Patient mit psychologischen Tricks gelenkt werden könne.

Im 3. Kapitel befaßt er sich mit der Euthanasie, die nichts anderes soll, als dem sterbenden Menschen («[dieser] ist eine heilige Person») das Scheiden aus dieser Welt zu erleichtern, wobei Vogel in pathetischen Tönen die unschätzbaren Verdienste eines wirklichen Arztes preist.

Im 4. Kapitel gibt er nun einen Entwurf eines diagnostischen Apparats: «Man hat zwar keineswegs diese Manier der Krankheitsforschung bisher gänzlich verkannt und versäumt; vielmehr sind die Prüfungsmittel, wovon ich hier reden will, von jedem guten Arzte einzeln längst angewandt worden. Man hat sie und ihre Gebrauchsart und Wirkung jedoch meines Wissens nie so genau erörtert, bestimmt und zusammengestellt, als ich es hier jetzt zu tun die Absicht habe.»

Die folgenden Kapitel sind der Diagnostik der Krankheiten der Kinder, Frauen und Greise gewidmet.

Im letzten Kapitel schließlich geht Vogel eingehend auf die Bedeutung der patholigischen Physiognomik, insbesondere der Augen ein. Damit greift er eine Strömung auf, die von Lavater neu belebt worden ist, wandelt sie aber für seine Zwecke ab. K.H.Baumgärtner erfüllte unwissentlich einen Wunsch Vogels (114) nach der bildlichen Darstellung als Ergänzung der unzureichenden Worte und ließ seine reich illustrierte Krankenphysiognomik (Stuttgart 1839 mit Atlas von 72 illuminierten Porträts) erscheinen.

Es ist naheliegend, zu prüfen, inwieweit sich, verglichen mit dem Krankenexamen, in den Allgemeinen medicinisch diagnostischen Untersuchungen Fortschritte der Medizin widerspiegeln, ob also Entwicklungen von 1796 bis 1824/31 erkennbar werden. Die beiden denkwürdigsten Errungenschaften sind zweifellos die von Corvisart neu belebte Perkussion und die Auskultation. Die Perkussion hat Vogel schon vor Corvisart angewandt, wenn er sie auch in den späteren Schriften ungleich stärker hervorhebt. $\mathrm{Da} \beta$ die mittelbare Auskultation 1796 nirgends erwähnt wird, versteht sich von selbst.

In anderen Bereichen Sicheres auszusagen fällt schwer. Beide Werke enthalten ungefähr dieselbe Zielsetzung, in beiden stehen sowohl psychologische, ethische und philosophische, wie auch physikalisch praktische Fragen zur Diskussion. Die Tatsache, daß in den Allgemeinen medicinisch diagnostischen Untersuchungen ein langer Paragraph der Erläuterung des diagnostischen Instrumentariums gewidmet ist, ist deshalb nicht wegweisend, weil Sonden, Spiegel, Katheter und andere vorwiegend chirur- 
gische Gegenstände ja schon längst bekannt waren und hier nur der Vollständigkeit halber aufgezählt sind.

Die Leistungen dieser Epoche bestanden in der Bestimmung und Abgrenzung einzelner Krankheiten und damit auch der Begründung verschiedener Spezialfächer, wegbereitend für spätere Jahrzehnte. Aber für wesentliche Schritte in der naturwissenschaftlich-physikalischen oder gar chemischen Diagnostik war es noch zu früh.

Das Versprechen in der Vorrede des Krankenexamens hat Vogel sicher gehalten, seine Ausführungen sind später umfangreicher und detaillierter geworden. Wie aber doch die meisten Punkte schon im Krankenexamen berührt sind, zeigen die Zitatangaben.

II. VORAUSSETZUNGEN FüR DIE ÄRZTLICHE BERUFSFÜHRUNG

\section{Das Arzt-Ideal}

Vogel mißt dem Zusammentreffen der nötigen Eigenschaften, die einen Menschen als Arzt qualifizieren, entscheidende Bedeutung zu. Mißerfolge seien leider nur zu oft auf ärztliches Ungenügen zurückzuführen. «[Diese] Eigenschaften sind in der That großentheils durch kein Studium und keine Anstrengung zu erreichen, sondern in angeborenen Vorzügen und Kräften des Geistes und besonderen Gemüthsbeschaffenheiten und Tugenden gegründet, welche zum Theil allerdings durch Erziehung, Beyspiel und Leitung, einer großen Ausbildung fähig sind» (89). Die caritativen, humanen Eigenschaften müßten dem Arzt in die Wiege gelegt worden sein, die Umgangsformen im weitesten Sinne seien dagegen weitgehend erlernbar.

«Der Charakter des Arztes spielt für die Diagnose eine mindestens so bedeutungsvolle Rolle wie die Sachkenntnis» (Hegglin 1953) (22).

Unbestechliche Beobachtungsgabe ist nach Vogel unabdingbar und würde durch ungebührliche Bevorzugung einer Lieblingswissenschaft oder gar unerwiesener Theorien beeinträchtigt. Weil selbstverständlich, stünden die vollständigen Kenntnisse der pathologischen, semiotischen und ätiologischen Erscheinungen außer Diskussion. Das Krankenexamen als Quelle und Stützte der Diagnose könne nur mit den erwähnten Eigenschaften erfolgreich sein. Es fehlt Vogel nicht an der nötigen Skepsis bei so hoch gestecktem Ziel: «Ob es solche ausgebildete Ideale von Ärzten in der Natur wirklich auch gebe, ist freylich eine andere Frage. In meinem langen Leben habe ich nur wenig Gelegenheit gehabt, Beyspiele kennenzulernen, die dem schon oft entworfenen Bilde nahe kamen» (89). 
Mit gehöriger Überlegenheit beschließt Vogel diesen Paragraphen: «Nur seltene Genies bringen es bey fortdauerndem Fleiße dahin, daß sie nach einigen Jahren kümmerlicher Praxis immer mehr begreifen, wie viel sie nicht wissen, und daß ein höchst wichtiger Theil der Kunst darin besteht, zu wissen, was man nicht thun dürfe.» (!) (89).

Was der Diagnose Leben, Wert und Sicherheit gebe, ist das «Savoir faire». Ohne dieses sei die Diagnostik ein leeres Wortspiel, «eine camera obscura» (105).

Was ist Savoir faire? Vogel hat darüber 1796 in Hufelands Journal eine längere Abhandlung geschrieben: «Zum Wohlgelingen einer jeden Handlung gehört, mehr oder weniger, eine gewisse Methode und Manier, diese Handlung zu verrichten. Darum ist das Resultat, wenn zwey ... Menschen dasselbe thun, oft so verschieden. Der Ausdruck savoir faire bedeutet, etwas so zu machen, daß es so gut als möglich gelingt. Es lehrt den Arzt, in der Behandlung der Kranken und ihrer Krankheiten alles so einzurichten, zu lenken und zu machen, daß die Absicht, sofern sie erreichbar ist, auf die günstigste und beste Weise erreicht wird. Sehr viel wichtiges savoir faire liegt vor allen Dingen in der Kunst des Arztes das Zutrauen seiner Kranken zu gewinnen und zu erhalten» (34). «Ohne dasselbe wird der Arzt bei jeder Gelegenheit getäuscht, übel berichtet und mißverstanden. Ohne dasselbe werden die treffendsten Maßregeln in ihrer Anwendung mißlingen. Sein Vertrauen, sein Glück, seine Ehre hängen davon ab» (104).

Das Savoir faire beinhaltet auch gewisse Kniffe und Notlügen, was Vogel einige Vorwürfe eingetragen hat. Er, vernünftig wie immer, rechtfertigt sich folgendermaßen: «Das savoir faire ist eben darum berechtigt, die Wahrheit zuweilen zu verbergen, sich dieser oder jener Maaßregel zu bedienen, um eine Grille, ein Vorurtheil, eine falsche Vorstellungsart eines Kranken zu verbessern, oder auch zu seinem Vortheile zu benutzen, mancher Krankheit, mancher Arzney einen andern Namen zu geben, mit einem Wort, jenen Weg, welcher zu dem vorgesteckten Ziele, nemlich dem Wohl des Kranken, ohne Verletzung einer wahren Pflicht, am schnellsten und sichersten führt, mit festen Schritten zu gehen» (35).

Das Entscheidende in der Medizin sei die Beobachtung, über deren Notwendigkeit zu schreiben Vogel völlig überflüssig erscheint: ars medica est tota in observationibus (Baglivi) (101, vgl. 49). Die dazu erforderlichen Eigenschaften hingegen könnten nie erschöpfend genug besprochen werden. Voraussetzung sei eine möglichst objekive, vorurteilsfreie Haltung; umfassende Kenntnis der verschiedensten Krankheiten, ihrer Verlaufs- und 
Spielarten erlaubten ein gezieltes Suchen und die so folgenschwere Trennung des Wesentlichen vom Zufälligen. So unvollständig auch die Kenntnis der ätiologischen Zusammenhänge noch sei, so gebe es doch nichts Aufschlußreicheres, als die verschiedensten Einflüsse zu beachten und nach Ursache und Wirkung getrennt zu bewerten (102). Täuschungen und Unwahrheiten von seiten der Kranken und der Umgebung seien besonders zu vermeiden. Gerade in großen Städten und Hospitälern sei die große Zahl der Patienten und die beengenden Verhältnisse eine Gefahr für die seriöse Diagnostik, worunter die armen Kranken verständlicherweise am meisten zu leiden hätten (103). Die großen Spitäler werden bezeichnenderweise erst 1831 erwähnt, nachdem die «Spitalmedizin» (Ackerknecht) aufgeblüht ist.

Das Savoir faire umschreibt also das den individuellen Gegebenheiten angepaßte, differenzierte psychologische Vorgehen des Arztes.

Ein eigenes Kapitel seiner Allgemeinen medicinisch diagnostischen Untersuchungen widmet Vogel der Kunst, das Sterben zu erleichtern. Auch er weiß, daß gerade erfahrene Ärzte den rätselhaften Kräften des Glaubens an Heilung große Bedeutung beimessen (106, vgl. 45). Ein für die Zeit typischer ethischer Hinweis darf hier nicht fehlen (vgl. 12, 13, 38): «Seine [des Arztes] Abneigung, solche Kranken, deren Cur er einmal übernommen hat, mit welchen er aber gar nicht fortkommen zu können glaubt, zu dienen, bleibt sehr natürlich; aber die Gründe, wodurch er sich wirklich bewegen lassen zu dürfen meynt, jener Abneigung Gehör zu geben, scheinen mir falsch, und den rechten Gesichtspunkt zu verfehlen. Er scheint sein Ich am Ende allen andern Betrachtungen vorzuziehen. Und dies darf niemand weniger als der Arzt, wenn er am Krankenbette sitzt» (34).

\section{Kenntnis des Patienten}

Auf langen Seiten erörtert Vogel, wie individuell Krankheiten verliefen und wie sehr sie von Alter, Geschlecht, Konstitution, Beruf, Ehe, sozialem Stand, Klima, Wohnung, Lebensführung, Gewohnheiten, Ernährung, Temperament, Charakter und Intellekt beeinflußt würden (60).

Sehr wichtig ist ihm die Physiognomik, die ja eine unerschöpfliche Vielfalt an geheimen Veränderungen aufzuzeigen vermag (110). "Alle Theile des Gesichts haben ihren Antheil an der Physiognomie, und fast keine Krankheit hat nicht Einfluß auf sie» (111). «Wie viele Krankheiten des Kopfs und seiner Theile, der Brust, des Unterleibs, der Säfte, der Nerven, 
der Seele, verrathen sich in diesem Spiegel!»(57). Es folgt eine ausführliche Beschreibung der Muskeln, Nerven und anderer Strukturen, die für die Physiognomie verantwortlich sind. Auf eine erschöpfende pathologische Physiognomik muß er verzichten, weil er dann eben auch eine beinahe vollständige Semiotik schreiben müßte (vgl. 111).

Er weiß viele richtige Zusammenhänge, die zum Teil seit Hippokrates bekannt sind: die Folgen eines cerebrovasculären Insultes wie Facialisparese, Aphasie, Hemiplegie usw. sind ihm bekannt; Meningismus bei Meningitis oder Encephalitis (36), eine ganze Reihe psychiatrischer Erkrankungen, Nervenleiden wie Epilepsie und Chorea, Tetanus mit seinem risus sardonicus, Lungen- und Herzkrankheiten rufen alle einen spezifischen Gesichtsausdruck hervor. Auch kennt er die facies abdominalis und hippocratica, Gelb-, Blau- und Bleichsucht und dank Loebenstein-Loebel (43) viele Details aus der Ophthalmologie: Mydriasis und Miosis, Photophobie, Strabismus, Nacht- und Schneeblindheit und das Phänomen der Mouches volantes.

Daß allein auf Grund des Gesichtsausdrucks eine Anhiebsdiagnose richtig sein kann, beweist in unserer Zeit Killian, der als Consiliarius bei einem Kranken mit Ileus-Verdacht schon im Türrahmen eine Lungenembolie diagnostizierte (37).

Alle diese breiten Kenntnisse über den kranken Menschen stehen im Dienste der Diagnose, helfen sie doch dem Arzt, nach kürzerem oder längerem Gespräch seine Untersuchungen gezielt vorzunehmen.

\section{Kenntnis der Krankheiten}

Bis dahin erscheint Vogels Wissen erstaunlicherwiese auch heute noch mehr oder weniger gültig, manche seiner ethischen aber auch physiognomischen Hinweise sogar beherzigenswert. Seine pathologischen Kenntnisse hingegen sind zwangsläufig zeitgebunden, sie haben die Morgagnischen zur Grundlage. Was Bichat als Bindeglied zwischen der Organpatholigie und Virchows Zellularpathologie mit seiner Gewebelehre geleistet hat, war ihm nicht aufgegangen, konnte ja wohl auch erst vom Gesichtspunkt der Virchowschen Erfolge aus als Fortschritt gedeutet werden.

Es war nicht Vogels Ziel, ein Handbuch der Semiotik, die die gefundenen Symptome einer bestimmten Krankheit zuordnet, oder der Pathologie, also eine allgemeine Krankheitslehre zu schreiben, sondern er wollte beschreiben, wie man Symptome suchen muß und entdecken kann; deshalb 
lassen sich nur hie und da Angaben finden, die von seiner Krankheitsauffassung Zeugnis ablegen. Aber so spärlich diese Stellen sind, so läßt sich doch daraus ableiten, welcher Art seine ätiologischen Ansichten sind. Auch hier macht er sich keiner Einseitigkeit schuldig. Einmal ist ein im Körper zirkulierendes Gift, ein andermal sind abnorme vegetative Nerven oder auch lokale Prozesse für eine Krankheit verantwortlich. Einen großen Platz nehmen die Erkältungen und Ausdünstungen ein (92). Die meisten, heute als bakterielle oder virale Infektionen bezeichneten Krankheiten führt er auf Erkältung zurück, manchmal mit phantastisch anmutenden Inkubationszeiten. Beeindruckt hat ihn die Kaempfsche Infarktlehre (93), er ist aber therapeutisch mit Klistieren wesentlich zurückhaltender. (Johann Kaempf [1726-1787] verdankt seinen Ruf der Theorie, daß gerade die hartnäckigsten Krankheiten auf blutige und fäkale Anschoppungen im Darm, eben auf ,Infarkte" zurückzuführen seien. Er hat also eine alte, schlechte Methode durch eine neue Erklärung aktualisiert. Seine Therapie bestand dann folgerichtig in einer erschöpfenden, wenn nicht gar tödlichen Zahl von Klistieren [20].)

Wichtig sind Vogel die Krankheiten, die wir heute den hormonell aktiven Lebensperioden zuordnen: «Es ist die Rede von den Krankheitsursachen, welche in den Entwicklungszuständen des menschlichen Körpers ... gegründet sind. Die aus dieser Quelle entspringenden krankhaften Erscheinungen sind so verschieden und mannigfaltig, daß sie leicht verkannt und falsch beurtheilt werden können. Nicht allein der Körper leidet daher eine Menge von Übeln, die zum Theil von ganz entgegengesetzter Natur sind, sondern auch die Psyche wird dadurch zu ganz verkehrten und abnormen Richtungen bestimmt und verleitet» (97). Es interessieren hier nicht die leib-seelischen Zusammenhänge, sondern die auch an andern Stellen (61) näher erläuterten Krankheiten während der Dentitionsperiode, Pubertät und Menopause. Die aufgezählten Krankheiten sind so unspezifisch, daß sie unsere Erwartungen in keiner Weise erfüllen können: «Entzündungen, Blutflüsse, Wahnsinn, Melancholie, acute Krankheiten aller Art, Hirnwassersucht» usw. (98).

Ist die funktionelle Beeinträchtigung mit dem morphologischen Befund erklärbar (z.B. Blasenausgangsstenose [91]), so bietet eine solche Erklärung dem rational beobachtenden Vogel keine Schwierigkeit. «Ich setze hinzu, daß auch die Untersuchung mit dem Finger durch den After nicht unterlassen werden dürfe. Wenn sich eine dem Finger darbietende Geschwulst der von Urin ausgedehnten Blase vorhanden ist, so wird der 
Druck darauf mit dem Finger einen Ausfluß des Urins zur Folge haben, und die Sache klar machen, vorausgesetzt, daß der Weg durch den Blasenhals und die Harnröhre offen und frey ist » (91). Ist aber eine Affektion nicht zu deuten, so kann er sich manchmal gewissen Spekulationen nicht verschließen (z. B. Blutanhäufungen im Kopf [95] oder ein Infarkt im Unterleib).

III. DIE EIGENTLIGHE DIAGNOSE

Es ist eigentlich erstaunlich, welches die Motive waren, die Diagnostik ständig zu verbessern zu suchen. Verglichen mit den heutigen Möglichkeiten erscheint die damalige Therapie mehr als bescheiden. Abgesehen von einigen wenigen wirksamen Heilmitteln wie etwa Digitalis, das Withering (1741-1799) von der Volksmedizin übernommen hatte (21), erschöpfte sie sich doch in Aderlaß, Opium und Klistieren. Das ist allerdings die heutige, historische Sicht, die seit Skoda als therapeutischer Skeptizismus bezeichnet wird. Damals aber war der Arzneimittelschatz oder was als solcher galt, riesig: es gab neben Chinarinde und Quecksilber Tonica, Antispasmodica, Emetica und vieles andere.

Für diesen Verbesserungswillen ist wohl einerseits der menschliche Drang, immer alles noch besser und genauer kennenzulernen, verantwortlich, andererseits wollte man möglichst genaue Prognosen geben können. Diese schon in der Antike wichtige Aufgabe der Medizin war auch zu Vogels Zeit nicht geschmälert: «Je länger das Vorspiel gedauert hat, an desto tiefer greifende und fester sitzende Ursachen muß man denken, und desto weniger hat man zu einer schnellen Abhelfung der Krankheit sich Hoffnung zu machen. Es lehrt daher von einer Seite Vorsicht in der Prognosis, und von der andern Behutsamkeit in der Cur» (66).

\section{Anamnese}

Vorausschickend mag zitiert werden, was Hegglin im Jahre 1953 über die Anamnese schreibt: «Die Erhebung der Vorgeschichte ist integrierender Bestandteil der ärztlichen Kunst, die kaum erlernbar ist ... Einige Hinweise technischer Art sind aber vielleicht nützlich: es ist ... wesentlich, daß der Patient seine Beschwerden frei schildern kann ... Der Kontakt zwischen Arzt und Krankem wird am raschesten hergestellt, wenn das den 
Patienten am meisten bewegende gegenwärige Leiden zuerst besprochen und erst später auf die früheren Krankheiten und die Familienanamnese ... eingegangen wird» (22).

Ganz analog heißt es bei Vogel: «Es würde sehr seltsam seyn, wenn sich der Arzt mit seinen Fragen durchaus immer wollte an eine gewisse Ordnung binden. Dies ist oft ganz unthunlich. Er muß sich in der Regel zunächst bey dem aufhalten, womit ihm der Kranke zuerst entgegen kommt» (55). Werfe der Kranke jedoch alles zu sehr untereinander, so sei zur Erleichterung und Beschleunigung ein striktes Fragen und Antworten angebracht.

Die Familienanamnese solle erbliche Dispositionen aufdecken, und die Kenntnis früherer Krankheiten und ihrer Behandlung ließe allfällige Idiosynkrasien erkennen (59).

Bei der persönlichen Anamnese seien nun alle Faktoren zu erforschen, die die individuelle Persönlichkeit des Kranken charakterisierten, wie Alter, Beruf, sozialer Stand, Gewohnheiten usw. (58).

Die Aufklärung des jetzigen Leidens müsse den Stellenwert der einzelnen Symptome offenbaren, was ja eine der wichtigsten Entscheidungen für die Diagnose ist (vgl. 44).

Vogel kennt das Phänomen der Immunität nach bestimmten Krankheiten wie Pocken und Masern und kann deshalb diese auf Grund der Anamnese ausschließen (63), was auch heute noch, wenn auch nicht in jedem Falle, richtig wäre. Er weist auf die Bedeutung spezifischer Prodromalbeschwerden hin: «Manche Krankheiten kündigen sich bey einigen Menschen durch gewisse Vorläufer an. Aus ähnlichen Vorläufern darf man also dieselben Krankheiten vermuthen » (64). Er betont aber die Schwierigkeiten bei ungenauen Angaben über die Zustände, die, vor langer Zeit erlitten, vergessen sind (65).

\section{Status praesens}

a) Inspektion

Unter den Begriff der Inspektion fällt nicht nur der klinische Blick und was bei der Physiognomik angedeutet wurde, also das beinahe intuitive Erfassen desssen, was auf den ersten Blick sichtbar ist, sondern auch das minutiöse Anschauen besonders derjenigen Körperstellen, die ein Leiden hervorrufen. «Die Mittel dazu sind die nöthige Helligkeit, eine bequeme Stellung des Bettes, um den Kranken von allen Seiten betrachten und behandeln zu können, Entfernung aller nicht unentberlichen Zuschauer 
usw.» (88). Sogleich werden auch wieder die Schwierigkeiten betont: «Manche Mängel und Schäden am Körper, zumahl beim weiblichen Geschlechte, werden geflissentlich geheim gehalten, z. B. ein Bruch, ein Prolapsus, eine Flechte, ein Ausfluß u. dgl. an verborgenen Orten» (88).

Man muß sich klar sein, daß die Patienten aus Schamhaftigkeit im allgemeinen dem untersuchenden Arzt ihren entblößten Körper nur ungern gezeigt haben. Bei der Palpation weist Vogel darauf hin, daß man das Hemd des Kranken anspannen müsse, um sich nicht durch getastete Falten irreführen zu lassen (96). Mit dem «Savoir faire» freilich bringe man die allermeisten Patienten dazu, sich auch an unangenehmen und peinlichen Stellen untersuchen zu lassen.

Der Gesichtsausdruck verrät am schnellsten und sichersten den Allgemeinzustand des Patienten. Während des anamnestischen Gesprächs wird sich dann der Arzt darüber klar, worauf er sich bei der Statuserhebung zu konzentrieren hat, ohne deswegen auf die systematische Durchuntersuchung zu verzichten. Denn oft entdeckt der Arzt «allerley Verunstaltungen, Geschwülste, Oberbeine usw., die der Kranke zufällig oder absichtlich verschweigen kann»(56). Schon aus der Lage, Stellung und eventuell dem Gang kann der Arzt schon vor der Anamnese mancherlei vermuten (62). Auch die Haut ist ein offensichtlicher und oft befallener Symptomträger. «Man untersucht sie durch das Gesicht und Gefühl. Man muß aber nicht bloß die Haut der gewöhnlich entblößten Theile untersuchen, sondern auch, so oft das Mindeste daraus klar werden kann, der Brust, des Unterleibes, des Rückens, der Schenkel und Füße, der Arme usw. Oft findet sich unvermittelt ein Ausschlag, eine locale Kälte, Hitze, Geschwulst, Röthe, ein Schweiß an einzelnen Theilen, eine Narbe, oder andere Dinge» (84). "An der Haut erforscht man außerdem überhaupt ihre Trockenheit, Humidität, Weichheit, Härte, Farbe, widernatürliche Empfindungen, als Jucken, Kribbeln, Brennen, Sprödigkeit, Reinheit, Wärme, Ausdehnung usw.» (85). Auch hier geht Vogel nicht auf die Bedeutung der Symptome ein.

Die Nägel können für die Semiotik wichtige Veränderungen zeigen (86). Ebenso ist die Untersuchung der Haare nicht immer ganz gleichgültig (74).

Der Bau der Brust ist besonders bei Lungenkrankheiten genau zu berücksichtigen. «Man sieht und fühlt Ödem, Emphysem, Geschwülste anderer Art ... (77). «Man sieht, ob die Brust platt, eingedrückt, verschoben und verunstaltet ist» (76). Hinweise auf Asymmetrien und Veränderungen, die von der inspiratorischen oder exspiratorischen Atemlage abhängen, 
finden sich im Kapitel «Diagnostik der Kinderkrankheiten»: «Bei der Respiration ist besonders noch zu bemerken, ob sich die Brust oder der Unterleib mehr, und wie, gleichmäßig oder ungleich, dabei hebt, zu schnell, langsam, unterbrochen ...» (109).

Dem Abdomen legt Vogel eine überragende Bedeutung bei: «Im Unterleibe liegen die Keime der Krankheiten, des Wohls und Wehs, des Glücks und Unglücks unzähliger Menschen» (79). Noch Beethoven glaubte, seine Taubheit sei auf ein Übel des Unterleibes zurückzuführen!

«Erst besieht man ihn (den Unterleib) überall äußerlich. Man bemerkt die Ausdehnung oder Eingezogenheit, die gleiche oder ungleiche Form, die Farbe und sonstige äußere Beschaffenheit der Haut, Ausschläge, Brüche, andere Geschwülste, Bewegungen im Leibe. Die Veränderungen der Ausdehnung muß man, wenn es darauf ankommt, eine jede geringe Abnahme oder Zunahme zu wissen, wie z.B. in der Wassersucht, durch messen, vermittelst eines umzulegenden Bandes, erforschen, und zwar muß dies des morgens nüchtern geschehen. Nöthig ist auch, den Unterleib in jeder Lage, auf den Seiten, dem Rücken, im Stehen zu beschauen» (80).

b) Palpation

Zusammen mit Anamnese und Inspektion bietet die Palpation eine aussagekräftige Untersuchungsmethode für das Abdomen: «Das Gefühl des Arztes ... entdeckt ... Spannung, gleiche oder ungleiche, glatte oder unebene Härten, Schmerzen und ihren Sitz, und Unempfindlichkeit, verborgene Geschwülste, Klopfen, Kälte, Hitze, Luft oder Wasser im Unterleib» (81). Wie wir noch heute, stellt Vogel den Ascites mit Hilfe der Undulation fest (82).

Er gibt genaue Stellungen des Patienten für die Abdominaluntersuchung an: im Liegen, Brust und Beine leicht angewinkelt; im Stehen, wobei der Oberkörper nach vorne gebeugt werden soll; und die Knie-EllenbogenLage: «die Leber, die Milz, das Pankreas, das Gekröse kommen auf diese Weise der in den Prächordien, Hypochondrien und tiefer in den Unterleib greifenden Hand näher» (82). Dabei müsse die Bauchmuskulatur immer erschlafft sein. Das Wesentliche bei diesen Untersuchungen sei immer, daß die gefundenen Symptome mit dem Befund der andẹren Methoden übereinstimmten (96).

Die digitale Palpation des Rektums weiß Vogel erxtaunlich vielseitig zu verwenden: «der Grund von manchen Leibesverstopfungen läßt sich 
nicht anders ausfindig machen, als daß man in den Mastdarm hineinfühlt. Es entdecken sich hier dann Verhärtungen, Verengerungen ... Auch erforscht man auf diesem Wege manche Krankheiten der Harnblase, der Gebärmutter» (83, vgl. 91).

Im Zusammenhang mit der Inspektion erwähnt Vogel häufig, daß der optische Eindruck mit dem Gefühl oft präzisiert werden könne, z.B. Ödeme, die Haut (s.o.S. 183).

Eine Untersuchungsmethode, die heute, weil zu grob, höchstens noch zum Hervorrufen des «Loslaß-Schmerzes» bei umschriebener Peritonitis verwendet wird, ist die Impression. Vogel dient sie vorwiegend dazu, die Pneumonie von der Pleuritis zu unterscheiden, indem bei der Pneumonie bei Druck auf das Epigastrium «Beklemmung, Husten, beschränktes Athemholen » (94) entstünden, bei der Pleuritis aber nicht. Ebenso könne Wassersucht des Herzbeutels erkannt werden, wenn beim Eindrücken des Epigastriums und somit Komprimieren des Herzens «gählinges Herzklopfen, beschleunigter Puls» (99) aufträten.

c) Puls

Das Fühlen des Pulses darf bei der Krankenuntersuchung nie fehlen, zuweilen hat er entscheidende Bedeutung: «Ich verließ einen Mann in vollem Fieber mit heftigen Schmerzen ... und Durchfall. Der Puls that wenigstens 100 Schläge in der Minute. Den andern Tag fand ich ihn nach einer schlaflos zugebrachten Nacht schwächer und leidender ... Ich vermuthete nichts gewisser, als einen schwächern, geschwindern Puls, wurde aber durch die beträchtliche Verminderung der Zahl der Pulsschläge ungemein überrascht. Ich war nun fest überzeugt, ... der Kranke gehe seiner Besserung entgegen, wie sich auch noch an diesem Tage deutlicher zeigte» (71).

Aber seine Skepsis verbietet Vogel, den Puls in seiner semiotischen Bedeutung zu überschätzen: «so trügend der Puls oft ist ...» (71). Aus dem Puls könne man mit der gebotenen Vorsicht meist lediglich allgemeine Schlüsse ziehen, etwa «das Maaß der Lebenskräfte, Reize, Krämpfe, Fieber, Hindernisse in der Circulation, Druck und Affection des Gehirns» (70). «Aber es giebt keine Krankheit und keinen Krankheitszustand, welchem eine einzelne Pulsart besonders und ausschließend eigen wäre» (72).

Wie man den Puls fühlen soll, ist offenbar zu selbstverständlich, um hier exakt beschrieben zu werden; dies wurde auch in zahlreichen speziellen Monographien und semiotischen Lehrbüchern ausführlich dargelegt. 
Es findet sich lediglich die Angabe, man müsse verschiedene Grade des Drucks mit dem Finger anwenden (68). Um sich genügend Gefühl an den Fingerspitzen zu bewahren, müsse man alles vermeiden, was sie hart und unempfindlich machen könne.

Die Aufregung beim Erwarten des ärztlichen Besuches beschleunige den Puls, deshalb solle man nicht gleich zu Beginn der Untersuchung den Puls fühlen, sondern erst warten, bis sich der Kranke etwas beruhigt habe und nur die Krankheit allein den Puls zu beeinflussen vermöchte (67).

Man kann den Puls nicht nur am Handgelenk, sondern auch am Herzen, am Hals, an den Schläfen, in der Kniekehle und an der Kinnlade tasten; auch soll man Seitenvergleiche anstellen (67).

Seine Tendenz zum Objektivieren und Quantifizieren läßt Vogel den Puls auch messen: «Wo es viel auf die Zahl der Pulse ankommt, muß man eine Secundenuhr zu Hilfe nehmen» (69).

\section{d) Perkussion}

Mit Recht bezeichnet man das Jahr 1761 als das Geburtsjahr der physikalischen Diagnostik, erschien doch damals Auenbruggers Inventum novum. Und doch ist das nicht ganz richtig, denn schon seit Hippokrates wurde das Abdomen bei Tympanie und Ascites perkutiert (40). So finden sich darüber auch bei Vogel Angaben (81). Das Schicksal der Thoraxperkurssion in ihren ersten Jahrzehnten ist bekannt: es erfolgte bald eine 2. Auflage des Inventum, aber die großen Kliniker taten sie mit der Bemerkung ab, schon Hippokrates habe von der Succussio gesprochen (welcher Vergleich jedoch unzulässig ist), und es wäre also nichts Neues, ein «Inventum novum antiquum», so Vogels Vater! (54). Erst durch Corvisart 1808 fand sie die gehörige Anerkennung und damit auch Verbreitung. Es erstaunt deshalb sehr, daß Vogel schon 1796 im Krankenexamen die Thoraxperkussion als wertvolle Methode hervorhebt: «man muß sie doch nie unterlassen» (78). Auch im späteren Werk wird das «Auenbrugger'sche Klopfen» wieder erwähnt (94). Nirgends steht etwas von der eigentlichen Technik oder den hervorrufbaren Schallqualitäten; Vogel hat es also vernünftigerweise unterlassen, die Originalliteratur abzuschreiben.

\section{e) Auskultation}

Vogel betont mehrmals, wie wichtig die Anstrengung aller Sinnesorgane für die Diagnostik sei - dazu gehört zweifellos auch das Gehör. Spricht er 
im Zusammenhang mit der Beobachtung des Atmens von hörbaren Tönen $(73,109)$ oder bei der Expektoration von «Geräusch auf der Brust» (87), so kann nicht entschieden werden, ob er diese auf Distanz oder mit der unmittelbaren Auskultation am Brustkorb selbst hört. Ob also Vogel diese Methode gekannt hat, bleibt zweifelhaft. Sicher aber hat sie Corvisart und sein Schüler Laennec gekannt, der ja zur Erfindung des Stethoskops dadurch äußerlich veranlaßt war, daß er wegen Korpulenz der Patientin die unmittelbare Auskultation nicht anwenden konnte. In den Allgemeinen medicinisch diagnostischen Untersuchungen heißt es dann in voller Anerkennung der neuen Methode: «Die Auenbrugger'sche durch Corvisart vorzüglich bestätigte, und nochmehr die Laennec'sche Methode, die Brustkrankheiten zu erkennen, würden hier ihre Dienste leisten» (94). Oder: «Das Laennec'sche Stethoskop verdient zur Erforschung der Brustkrankheiten ohnstreitig besondere Aufmerksamkeit» (100). Natürlich wußte Vogel auch mit dem Stethoskop die Herztöne zu hören, nicht zuletzt, um den sicheren Tod (112) vom Scheintod (113) zu unterscheiden.

Das Problem des Scheintodes hat gerade zu Vogels Zeit die Gemüter heftig erregt. Im Nachtrag zum Kapitel 3 der Allgemeinen medicinisch diagnostischen Untersuchungen sind zahlreiche Schriften genannt, die sich mit den sicheren Todeszeichen befassen, Kenntnisse, um den Scheintod ausschließen zu können. Gerade das Zeitalter der Aufklärung wollte zwischen dem geliebten Diesseits und dem unvermeidlichen Jenseits eine eindeutige Grenze haben (vgl. 46).

Bemerkenswert ist doch die Anpassungsfähigkeit Vogels, der mit über 70 bzw. 80 Jahren die so wesentliche Erweiterung der Diagnostik anerkennt. Diese Tatsache macht auch seinen Glauben und seine Überzeugung: «ars medica est tota in observationibus» (101) wirklich glaubwürdig und paßt schön zu seiner strikten Ablehnung der Naturphilosophie.

\section{f) Diagnostisches Instrumentarium}

Die chemische Diagnostik scheint aus heutiger Sicht noch sehr bescheiden. Nach den vorliegenden Angaben hat sie sich mit der Analyse der Harnkonkremente und der Bestimmung von Acidität bzw. Alkalinität der Sekrete und des Eiters zufrieden geben müssen (107). Aber immerhin, auch dies ist ein Zeichen dafür, wie Vogel jede rationale Möglichkeit aufgreift und sie dem Leser mit der Ermahnung zur Lektüre vorlegt, durch eigenes Beobachten und Üben Neues beizutragen. 
Vogel hat um 1825 die Herstellung eines «portativen Prüfungsetui» veranlaßt, worin die wichtigsten Instrumente enthalten sind $(108,113)$ : eine Linse, die der genaueren Inspektion dient; ein Zirkel, ein Maßstab und eine Meßbinde, die zur Abmessung verschiedener Distanzen verwendet werden sollen, besonders, um den Verlauf bei pathologischer Ab- oder $\mathrm{Zu}$ nahme, etwa bei Ascites, verfolgen zu können. Es sind auch einige Instrumente eher therapeutischer Bestimmung aufgezählt, etwa ein Messer, eine kleine Schere, eine Pincette und, merkwürdig, Akupunkturnadeln. Die Akupunktur wurde 1810 von einem französischen Arzt namens Berlioz in die westliche Praxis eingeführt und fand einige Verbreitung besonders in Frankreich und England. Vogel muß davon gehört oder gelesen und diese Methode für wichtig genug erachtet haben, sie hier zu erwähnen (17).

Außerdem empfielt er folgende Hilfsmittel: «eine Secundenuhr zur Bestimmung der Frequenz der Pulsschläge» (69); «ein Taschenthermometer zur Prüfung der Temperatur des Körpers». Auch dies ist wieder ein Hinweis, wie sorgsam Vogel alle diagnostischen Hilfsmittel, die der Zeit zur Verfügung gestanden haben, aufgriff und nicht wie seine Zeitgenossen vergaß: Boerhaave hatte gelegentlich Temperaturmessungen vorgenommen. Seine Schüler van Swieten und de Haen haben die Thermometrie an ihrem neuen Wirkungsort Wien eingeführt und sie stark vorangetrieben. Dann aber geriet sie bis zur Mitte des 19. Jahrhunderts in Vergessenheit, als sie Traube und Wunderlich wieder aufnahmen und der zweite ihr ganz neue Dimensionen eröffnet hat (47).

«Ein Schnepper, eine Lancette, ein Troicart», um durch das Ablassen des Inhalts von Abszessen, Geschwülsten und Wasseransammlungen Linderung der Schmerzen herbeiführen und zur Untersuchung gewinnen zu können, sind weitere Instrumente.

Vogel nennt einige Specula, die palpatorische Befunde zu erhärten vermögen, so das speculum uteri: «Es leistet, was die bloße Exploration nicht leisten kann, indem es namentlich z.B. krebshafte Zerstörungen des Uterus in Folge scirrhöser Verhärtungen zu erkennen gibt. Letztere kann der explorierende Finger wohl fühlen, aber nicht so die ersteren.» Mit dem heute als Rektoskop bezeichneten Instrument könne man carcinomatöse Veränderungen und Stenosen ermitteln.

Auch Spatel, Sonden und Katheter sind bei kasuistischen Berichten anzutreffen $(75,90)$.

«Die Piorry'sche Perkussionsscheibe oder Plessimeter, das von Piorry verbesserte Laennec'sche Stethoskop» sind Vogels Aufmerksamkeit auch 
nicht entgangen. Hier folgt die sehr aufschlußreiche Bemerkung: «Es wird nun wohl noch wenige Ärzte mehr geben, die dieses Instrument nicht kennen» (108).

Erwähnenswert ist auch eine Weiterentwicklung des Stethoskops, das Metroskop. Mit diesem Instrument solle man die Töne und Bewegungen hören können, die im Uterus stattfänden. Man könne nämlich die Schläge der Arterien vernehmen und so eine Placenta praevia entdecken. Man könne zuweilen auch davon die Herzschläge des Fötus unterscheiden (113, vgl. 15, 42).

Das Pneumometer war ein Instrument, das die Atemkapazität messen konnte (108).

Wie sehr steht doch all dies im Gegensatz zur deutschen Medizin des Jahrhundertbeginns! «Ein großer Teil nicht unbedeutender Ärzte (war) in Naturphilosophie und Brownianismus und damit in dem Glauben befangen, in der philosophischen Spekulation den sicheren Grund der Medizin gefunden zu haben» (19).

\section{HUFELAND ALS DIAGNOSTIKER - EIN VERGLEICH}

Sicher lohnt sich ein Vergleich der Vogelschen Diagnostik mit derjenigen eines Zeitgenossen, der sich auch von Theorien und Spekulationen hat freihalten können: Christoph Wilhelm Hufeland. Der um zwölf Jahre Jüngere hat in Jena und auch in Göttingen studiert. Nach dem Studium mußte er die Praxis seines beinahe erblindeten Vaters in Weimar übernehmen. Dank der Vermittlung Goethes wurde er 1793 Professor in Jena, wo seine Vorlesung über die Makrobiotik oder «die Kunst, das menschliche Leben zu verlängern » die sagenhafte $Z$ ahl von 500 Hörern anlockte.

Seine vernünftigen Einsichten besonders in die Heilkräfte der Natur ließen ihn zum heftigsten Gegner der Brownschen Lehre werden. 1801 ging er nach Berlin, gründete eine für die Armen unentgeltliche Poliklinik und wurde der erste Professor der Medizin an der 1810 gegründeten neuen Universität.

Er hat die Auffassung vom ärztlichen Künstlertum neu belebt: «Die Hauptsache in der Medizin besteht darin, das Urteil über die Krankheit und über das Heilverfahren aus dem Kranken heraus, nicht in den Kranken hinein zu tragen.» - «Jeder Kranke ist ein Tempel der Natur. Nahe dich ihm mit Ehrfurcht und Weihe, entferne von dir Leichtsinn, Selbstsucht 
und Gewissenlosigkeit, dann wird sie gnädig auf dich blicken und ihre Geheimnisse dir aufschließen » (cit. nach 16).

Als «Vermächtnis einer fünfzigjährigen Erfahrung» gab er 1836 ein Enchiridion medicum heraus, eine Anleitung zur medizinischen Praxis. Seine Indifferenz der physikalischen Diagnostik gegenüber, die ja hier hauptsächlich interessiert, ist bemerkenswert. Jahrzehnte waren doch schon vergangen seit Auenbruggers Inventum, auch seit der Wiederentdeckung durch Corvisart, seit Laennecs Traité, seit Corvisarts Ausspruch: «nollem esse medicus sine percussione et auscultatione»(!) (53). Noch war eben nur Paris und Wien, nicht aber auch Berlin führend in der neuen Medizin.

Zugegeben, Hufeland hat die Palpation der druckdolenten entzündeten Leber (26) und Milz (27) empfohlen, ebenso die Ascitespunktion (29) und die Rektalpalpation (32). Bei der Brust- und Lungenentzündung heißt es aber: «Man hat in neuern Zeiten auch die Zeichen durch das Gehör, mit Hülfe des Stethoskops oder der Perkussion, zur Diagnose der Brustkrankheiten, sehr empfohlen. Aber diese Zeichen sind sehr trüglich, und durch sie allein wird man nie das Dasein einer Entzündung entdecken, wenn man nicht die andern Zeichen zu Hülfe nimmt, die allein schon zur Diagnose hinreichen. Sie können also höchstens dazu dienen, über die Stelle der Entzündung einiges Licht zu erhalten, welches aber zur Indikation gar nichts hilft und auf die Bestimmung der Behandlung keinen Einfluß hat » (25).

Auch bei der Phthisis pulmonalis überwiegt die Skepsis: «Die Zeichen der Auskultation, Perkussion und des Stethoskop können als Hülfszeichen ... benutzt werden, aber nie zur Bestimmung der Diagnose im ganzen, da ja das Hören nicht einmal unterscheiden läßt, ob die röchelnde Materie Schleim oder Eiter ist» (28). Hätte er sich das Wissen Laennecs aneignen wollen, so hätte er niemals mit dieser Kausalität argumentieren können.

Beim Hydrothorax (30) und beim Hydrops pericardii (31) empfiehlt er die Auskultation, nicht aber die Perkussion.

Auch in späteren Jahrzehnten haben sich neue Methoden nicht sofort durchgesetzt. So erzählt W. Löffler in einem Aufsatz «100 Jahre Davos auf medizingeschichtlichem Hintergrund»(52) über die Anfänge der Radiologie: «Sogar noch 1920 hat es autoritativ vielfach geheißen: Wenn es hochkommt, so bestätigt das Röntgenbild den klinischen Befund.»

Der Puls hat für Hufeland noch eminente Bedeutung: «Der Arzt muß den Puls behandeln, wie der Virtuos sein Instrument ... Nur ein solcher 
Arzt wird Dinge in und durch den Puls entdecken, wovon ein anderer gar keine Ahnung hat» (23).

Ebenso stark hängt er an der Uroskopie. Er kann aus dem Urin ersehen, ob der Zustand des Patienten kritisch ist oder nicht, er unterscheidet nervöse, krampfhafte und gastrische Zustände (24). Erstaunlicherweise entsprechen auch seine physiologischen Vorstellungen weitgehend den antiken Lehrern. «[Der Urin] verdient also die größte Aufmerksamkeit des Arztes, die ihm bei den alten Ärzten in so hohem Grade zu Theil ward und jetzt viel zu sehr vernachlässigt wird» (vgl. 39).

Der Vergleich fällt somit recht enttäuschend und zu Ungunsten Hufelands aus; die ethische und psychologische Meisterschaft zeichnet beide aus, aber die überragenden Möglichkeiten der neuen Diagnostik hat der zwölf Jahre ältere Vogel viel entschiedener aufgegriffen.

Bedenkt man, daß Hufelands Buch während über zwanzig Jahren immer neue Auflagen erfahren hat, so ist es nicht verwunderlich, daß die zweite Hälfte des 19. Jahrhunderts von den Jahren bis etwa 1840 nur eine geringe Meinung gehabt hat. Wunderlich hat mit Vehemenz über diese Zeit geschrieben: «Nur im seltensten Fall kam die diagnostische Untersuchung auf handgreifliche und klare Antworten, sondern sie schloß mit nebelhaften, nicht weiter zu analysierenden und ebensowenig zu fassenden Begriffen» (117). - «Hatte die Naturphilosophie die Köpfe verdreht und den Sinn von der sogenannten gemeinen Wirklichkeit weggerissen, hatte die Erregungstheorie das Nachdenken in einem leeren Formalismus aufgehen lassen, so ist dem Eklekticimus die Verödung der deutschen Medicin zuzuschreiben. So kam es, daß in den ersten 30 Jahren des Jahrhunderts in keinem Lande eine schlechtere und schlaffere Medicin herrschte, als in Deutschland» (116). Den Universitätsbetrieb tut er noch energischer ab: «Nichtssagende Systematik mußte die Inhaltslosigkeit ersetzen. Wo noch ... der Unterricht lateinisch ertheilt wurde, ging er vollends in leerem Phrasenwesen auf» (116). Hufelands Journal selbst nennt er «[einen] Sammelplatz für alle schlaffe Erfahrung und [ein] Denkmal der sterilen Periode der deutschen Medicin» (115).

Wunderlichs enragierte Stellungsnahme ist verständlich, steht er doch am Anfang der exakten Naturwissenschaft, die unsere Welt verändert hat. Allein, heute sehen wir, daß dieser streng naturwissenschaftliche Weg in der Medizin auch nicht letztlich ans Ziel führt. So schreibt Bamm kritisch von der Überschätzung der zellularpathologischen Hypothese Virchows: «Das klinische Wissen der alten Ärzte vom Menschen als lebendigem Wesen 
ging weit über das hinaus, was der an der Leiche sich orientierenden Medizin zugänglich ist» (14).

Die von Wunderlich so heftig kritisierte Zeit Vogels, die noch wußte, was das Wort Seele bedeutet, hat Bereiche in der Medizin berücksichtigt, denen man sich erst in jüngster Zeit wieder zuzuwenden wagt. Sie Soziologie und die Psychologie versuchen heute im Dienste der Medizin die Seiten der menschlichen Existenz zu erforschen, die die exakte Naturwissenschaft vernachlässigt, weil sie ja den Menschen zu einer Arbeitshypothese hat degradieren müssen. Daß sie dabei Unglaubliches geleistet hat, steht außer Zweifel, aber ein Mißbehagen über ihre Erfolge ist auch nicht zu leugnen. Es liegt in der Natur der exakten Wissenschaft, jedes Subjekt, das sie untersuchen will, zu objektivieren und zu reduzieren, damit es methodisch überhaupt erfaßbar wird. Die Soziologie und die Psychologie erheben den Anspruch, exakte Wissenschaften zu sein. Also sind auch sie gezwungen, gewisse Aspekte zu vernachlässigen. Die Gefahr ihrer Forschung besteht darin, daß sie, wie schon die Naturwissenschaft vor ihnen, die moralischen und ethischen Aspekte der Heilkunde ausklammert. Der Philosoph wird sagen, daß diese niemals Gegenstand der wissenschaftlichen Forschung sein werden, sondern daß nur die persönlichen Qualitäten des Arztes ihre Integrierung in die Heilkunst gewährleisten können.

Dieser Gesichtspunkt nun führt unmittelbar zu Vogel und seinen im Kapitel «Das Arzt-Ideal» zitierten Aussagen zurück.

\section{Zusammenfassung}

Nach einem Überblick über Vogels Leben, die medizinhistorische Situation seines Zeitalters und seine diagnostischen Schriften verfolgt die vorliegende Arbeit das Ziel, den Gang der medizinischen Untersuchung zu Ende des 18. Jahrhunderts anhand der diagnostischen Anweisungen Vogels aufzuzeigen.

Eindrucksvoll ist seine Charakteristik der Qualitäten des idealen Arztes, die die Voraussetzung zu einer guten Berufsführung sind.

Daß Vogel als Rostocker Professor durchaus auf die Praxis bezogen war und das diagnostische Vorgehen so schildert, wie es sich bis heute im wesentlichen bewährt hat, kontrastiert mit der für die Medizin so verhängnisvollen Rolle der Naturphilosophie. Der leider einflußreiche Hufeland hält deshalb einem Vergleich mit Vogels Diagnostik nicht stand. Vogels über- 
zeugtes Eintreten für sinnvolle und brauchbare Methoden hat ihn veranlaßt, die Perkussion schon vor Corvisart und die Auskultation kurz nach ihrer Erfindung zu empfehlen.

\section{Literatur zur Biographie}

1 Brandenburger W., Physikus Dr. Samuel Gottlieb Vogel, der Vater des deutschen Seebades, in Öffentlicher Gesundheitsdienst (Stuttgart) 20 (1958) 3, 107-109.

2 Rohlfs H., Die medicinischen Classiker Deutschlands, 2. Abth., Stuttgart 1880: Samuel Gottlieb von Vogel, der Vater des deutschen Seebades, S. 66-126.

\section{Literatur zur allgemeinen Medizingeschichte}

3 Ackerknecht E.H., Kurze Geschichte der Medizin, 2. Auflage, Stuttgart 1959.

4 - Medicine at the Paris Hospital 1794-1848, Baltimore 1967.

5 - Medizin und Aufklärung, in Schweiz. med. Wschr. 89 (1959) 1, 20.

6 Aschoff, Diepgen, Goerke, Kurze Übersichtstabelle zur Geschichte der Medizin, 7. Auflage, Berlin 1960.

7 Diepgen P., Geschichte der Medizin, 2. Band, 1. Hälfte, Berlin 1951.

8 Garrison F.H., An Introduction to the History of Medicine, 4th ed., Philadelphia 1929, reprinted 1960.

9 ManN G., Medizin der Aufklärung, in Marburger Universitätsbund, Jahrbuch 1966/67, S. 321-332.

10 Sigerist H., Große Ärzte, 5. Auflage, München 1965.

11 Steudel J., Wesenszüge der Medizin des 18. Jahrhunderts, in Dtsch. med. J. 5 (1954) $3 / 4,81-86$.

\section{Anmerkungen}

12 Ackerknecht E.H., op. cit. sub no. 3, S.114.

13 - Zur Geschichte der medizinischen Ethik, in Praxis 53 (1964) 578-581.

14 Baмm P., Ex Ovo, Essays über die Medizin, Stuttgart 1956, Neuausgabe 1967, S. 93.

15 Bibliothèque universelle des sciences, belles-lettres et arts, tome 9, Genève 1818, S. 249 Fußnote.

16 Brauchle A., Naturheilkunde in Lebensbildern, Leipzig 1937, S.34-49.

17 Dechambre A. (Hrsg.), Dictionnaire encyclopédique des sciences médicales, $1^{\text {re }}$ série, 1. Band, Paris 1869, S.670-688.

18 Ebstein E., Der Rostocker Professor S. G. Vogel (1750-1837), ein ausgesprochener Gegner der Naturphilosophie, in Med. Klin. XV (1919) 50, 1299-1300.

19 Fischer H., Arzt und Humanismus, Zürich 1962: Die Krankheitsauffassung Friedrich von Hardenbergs (Novalis) 1772-1801, S. 257.

20 Garrison F.H., op. cit. sub no. 8, S. 315.

21 - ibid. S. 357.

22 Hegglin R., Differentialdiagnose innerer Krankheiten, 2. Auflage, Stuttgart 1953, S.22. 
23 Hufeland C.W., Enchiridion medicum oder Anleitung zur medizinischen Praxis, Berlin 1836, S. 30 .

24 - ibid. S. 48.

25 - ibid. S. 157.

26 - ibid. S. 171.

27 - ibid. S. 173.

28 - ibid. S. 319.

29 - ibid. S. 356.

30 - ibid. S. 360 .

31 - ibid. S. 361.

32 - ibid. S. 374.

33 - in Journal der Moden 1789, zit. nach K. JoEL, Wandlungen der Weltanschauung, Band 1, Tübingen 1928, S.635.

34 - (Hrsg.), Journal der practischen Arzneykunde und Wundarzneykunst, Jena, Band I, 3. St. (1796), S.295-297.

35 - ibid., Band VIII, 3. St. (1799), S. 85-87.

36 Killian H., Facies dolorosa, 3. Auflage, München 1967, S.68-69.

37 - ibid., S. 71.

38 Koelbing H.M., Die «Ärztliche Ethik» des Thomas Percival, in Schweiz. med. Wschr. 97 (1967) 22, 713-716.

39 - Der Urin im medizinischen Denken, VI. Documenta Geigy (Basel) 1967, 84-91.

40 LeSky E., Leopold Auenbrugger - Schüler van Swietens, in Dtsch. med. Wschr. 84 (1959) 22, 1017-1022.

41 - Cabanis und die Gewißheit der Heilkunde, in Gesnerus (Aarau) 11 (1954) 3/4, 173.

42 - Zur Geschichte diagnostischer Methoden I, Perkussion und Auskultation, Documenta Geigy (Basel) 1970.

43 Loebenstein-Loebel E., (1779-1819), Grundriß der Semiologie des Auges für Ärzte, Jena 1817.

44 NAEgeLi O., Differentialdiagnose in der inneren Medizin, Leipzig 1937, S.9-11.

45 Nissen R., Helle Blätter - dunkle Blätter, Stuttgart 1969: Das Wunder der Heilung, S. 73-78.

46 Patak M., Die Angst vor dem Scheintod in der 2. Hälfte des 18.Jahrhunderts, Diss., Zürich 1967.

47 Pontmann M.L., Histoire de la thermométrie clinique, in Méd. Hyg. (Genève) 27 (1969) 858, S.172-175.

48 Rотнsснuн K.E., Geschichte der Physiologie, Berlin 1953, S. 96.

49 Sigerist H., op. cit. sub no. 10, Giorgio Baglivi, S.151.

50 - ibid.: Thomas Sydenham, S. 159.

51 Sudhoff K., Das medizinische Zeitschriftenwesen in Deutschland bis zur Mitte des 19. Jahrhunderts, in Münch. med. Wschr. 50 (1903) 11, S.455-463.

52 Suter F. und H.Meyer (Herausgeber), Hundert Jahre Lungenkurort Davos, Bern 1966, S. 22.

53 Vierondt H., Kurzer Abriß der Perkussion und Auskultation, 3. Auflage, Tübingen 1890; Motto auf dem Titelblatt.

54 Vogel R.A., N. med. Bibl. 6 (1766), zit. nach 40. 
55 Vogel S. G., Das Krankenexamen oder allgemeine philosophisch medicinische Untersuchungen zur Erforschung der Krankheiten des menschlichen Körpers, Stendal 1796, $\S 16$.

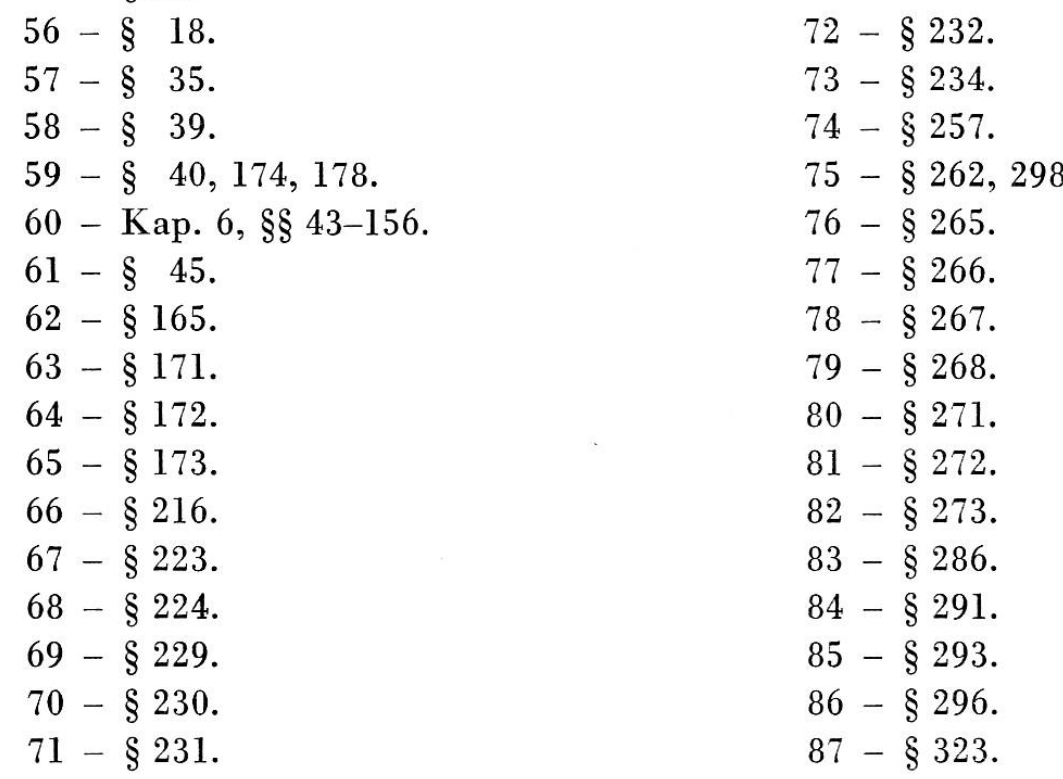

88 - Allgemeine medicinisch diagnostische Untersuchungen zur Erweiterung und Vervollkommnung seines Krankenexamens, Theil I, Stendal 1824, § 5.

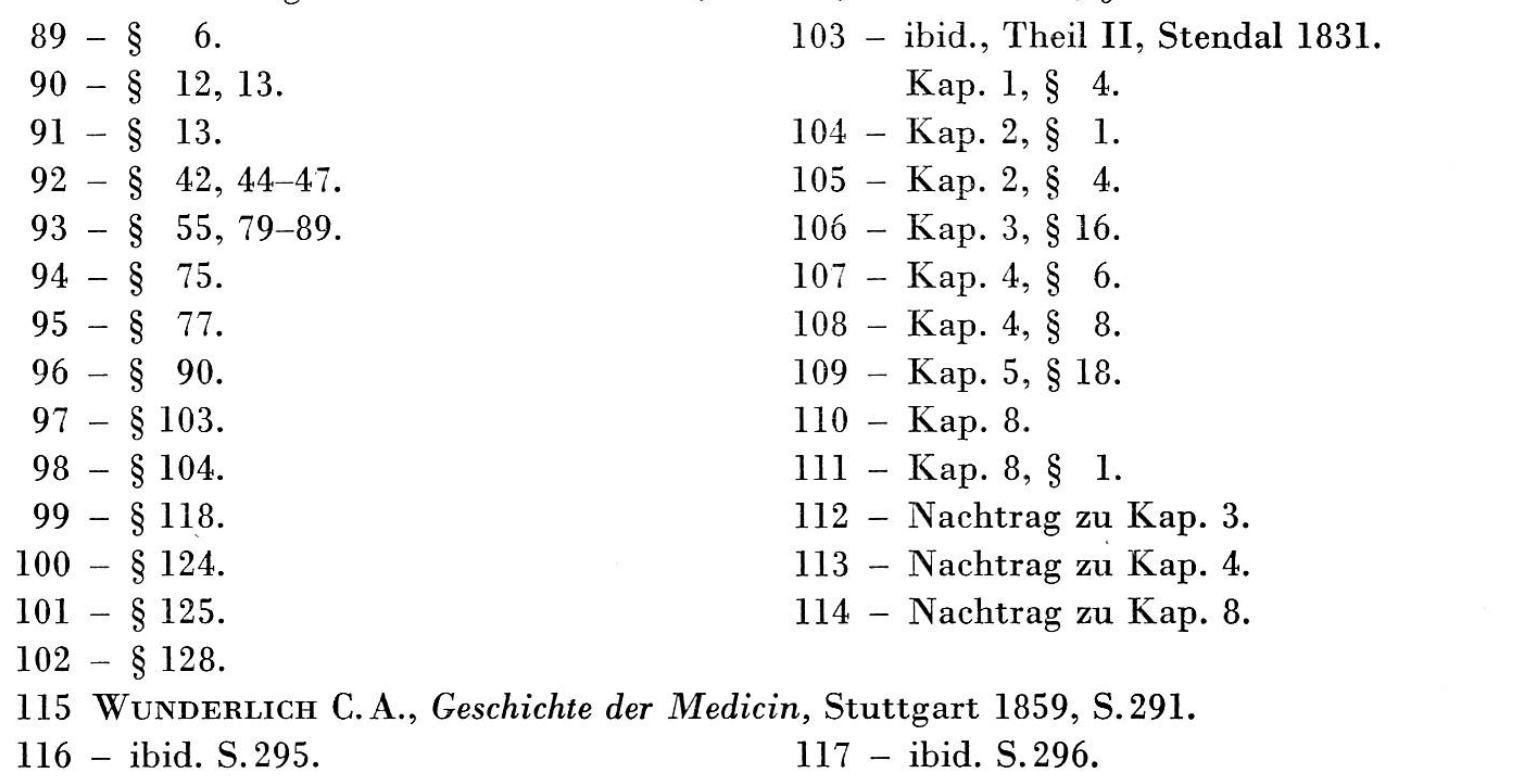

